\title{
Modeling Traffic Flow at an Urban Unsignalized Intersection
}

\author{
H. J. Ruskin and R. Wang \\ School of Computer Applications, Dublin City University, Dublin 9, Ireland \\ \{hruskin, rwang\} @compapp.dcu.ie
}

\begin{abstract}
This paper proposes a new way to study traffic flow at an urban unsignalised intersection, through detailed space considerations, using cellular automata (CA). Heterogeneity and inconsistency are simulated by incorporation of different categories of driver behaviour and reassignment of categories with given probabilities at each time step. The method is able to reproduce many features of urban traffic, for which gap-acceptance models are less appropriate. Capacities of the minor-stream in a TWSC intersection are found to depend on flow rates of major-streams, also changes with flow rate ratio $(\boldsymbol{F} \boldsymbol{R} \boldsymbol{R}=$ flow rate of near lane: flow rate of far lane). Hence flow rates corresponding to each stream must be distinguished. The relationship between the performance of intersections and other traffic flow parameters is also considered. Vehicle movements in this paper relate to left-side driving, such as found in UK/Ireland. However, rules are generally applicable.
\end{abstract}

Keywords: Modelling, cellular automata, unsignalised intersection, capacity, TWSC

\section{Introduction}

Two types of unsignalised intersections have been the main focus in modelling uncontrolled intersection flow. These are the two-way stop-controlled intersection (TWSC) and all-way stop-controlled intersection (AWSC). AWSC and TWSC are typical in North America and UK/ Ireland respectively. We focus on the latter here.

Performance measurements for TWSC have included capacity, (the maximum number of vehicles that can pass through an intersection from a given road), queuelength and delay. Both empirical and analytical methods have been used. The former includes Kimber's model [1] and the linear capacity model [2], while the most common analytical method uses the gap-acceptance criterion [3].

Cellular automata (CA) models provide an efficient way to model traffic flow on highway and urban networks, [4-6]. The CA model is designed to describe stochastic interaction between individual vehicles, independently of headway distribution and can be applied to most features of traffic flow, whether or not these can be described by a theoretical distribution. Features modelled may include multi-streams on the major road, heterogeneous vehicles (passenger and heavy vehicles), and intersections with or without flaring. 


\section{Background}

The basic assumption of gap-acceptance models is that the driver will enter the intersection when a safe opportunity or "gap" occurs in the traffic. Gaps are measured in time and correspond to headway, (defined as distance divided by speed). Critical gap and follow-up time are the two main parameters, where the critical gap is defined as the minimum time interval required for one minor-stream vehicle to enter the intersection. The follow-up time is the time span between two departing vehicles, under the condition of continuous queueing.

Gap-acceptance models are, however, unrealistic in assuming that drivers are consistent and homogenous [7, 8]. A consistent driver would be expected to behave in the same way in all similar situations, while in a homogenous population, all drivers have the same critical gap and are expected to behave uniformly [9]. In any simulation, therefore, driver type may differ and the critical gap for a particular driver should be represented by a stochastic distribution, Bottom and Ashworth [10].

In gap acceptance models, estimation of the critical gap has attracted much attention, with use of a mean critical gap also proposed [11-13]. Maximum likelihood estimation of the mean critical gap has been widely accepted [3, 12-15], but the basic assumption is still that all drivers are consistent.

Tian et al. [16] investigated the factors affecting critical gap and follow-up time, concluding that drivers use shorter critical gap at higher flow and delay conditions. Many other factors have also been found to affect critical gap [16-18], so that a critical value, obtained for any given situation, is unlikely to be generally applicable.

Further, gap-acceptance models have failed to consider conflicts between the two major-streams. When right-turning vehicles (for left-side driving) in the major-stream of a narrow road give way to straight-through vehicles from the opposing stream, a queue will form on the major-stream behind the subject vehicle, (i.e. turning-left and going-straight vehicles share the same lane). The headway distributions are affected so that original gap-acceptance criteria no longer apply.

At an unsignalised intersection in an urban network, adjacent intersections with traffic lights will have grouped the vehicles into a queue (or queues) during the red signal phases, and platoons will thus be present, (i.e. a filtering effect). The filtering of traffic flow by traffic signals has a significant impact on capacity and performance [19]. In particular, the gap-acceptance model can be applied only when no platoon is present [20]. Otherwise, no minor-stream vehicle can enter the intersection, as the mean headway within a platoon is supposed to be less than the critical gap. If traffic signal cycles are known and co-ordinated, the platoon pattern may be predictable. Otherwise, traditional gap-acceptance is not readily applied [20].

Headway distributions are also affected by traffic lights and in absence of these, platoon formation will occur due to the vehicle speeds. Further, critical gap is not easy to define and implement when several traffic streams are involved [3] and gapacceptance does not specifically allow for modelling directional flow [16].

A CA model is thus proposed, using analogous but more flexible methodology compared to gap acceptance, (e.g. spatial and temporal details of vehicle interactions can be described). This not only facilitates understanding of the interaction between the drivers, but can also be applied to situations for which headway distributions are insufficient to describe traffic flow. This paper considers combinations of available 
space on several major/minor streams and extends previous work on single conflicting flows [21].

A CA ring was firstly proposed for unsignalised intersections [5, 22]. All entry roads are "connected" on the ring. The car "on the ring" has priority over any new entry. However, there is no differentiation between the major and minor entry roads and all vehicles have equal priority to move into the ring (intersection), which compromises usual TWSC rules. A further CA model variant for intersections is described [23].

\section{Methodology}

A two-speed one-dimensional deterministic CA model, [5, 24, 25], is used to simulate interaction between drivers on the intersection only. The speed of a vehicle is either 0 or $1\left(v_{\max }=1\right)$, i.e. the vehicle can move only one cell in a time-step, ( 1 second for our model). The length of each cell corresponds to "average speed" on given intersection, e.g. length of $1 \mathrm{cell} \cong 13.9 \mathrm{~m}$ for speed of $50 \mathrm{~km} / \mathrm{h}$.

While multi-speed models [4] are somewhat similar, these have many features, which are superfluous for urban features, or to representation of driver behaviour [5]. Moreover, vehicle dynamics are often less important than driver interactions in simulating queue formation in urban networks [22].

A multi-speed one-dimensional deterministic CA model $[4,26]$ is used here to model the traffic flow on the straight roads only (intersection excluded). Speed of vehicles is 0,1 or $2\left(v_{\max }=2\right)$, corresponding to speed of $0,25 \mathrm{~km} / \mathrm{h}$ and $50 \mathrm{~km} / \mathrm{h}$. Length of 1 cell $\cong 7 \mathrm{~m}$. The difference between the $\mathrm{t}$

The update rules for each time-step are:

- Vehicle moves $v(=0$ or 1:two-speed CA or 0,1 or $2:$ multi-speed CA ) cells ahead

- $\quad$ Find the number of empty cells ahead $=E$

- If $v<E$ and $v<v_{\max }$, then $v$ increased to $v+1$

- If $v \geq E$, then $v=E$

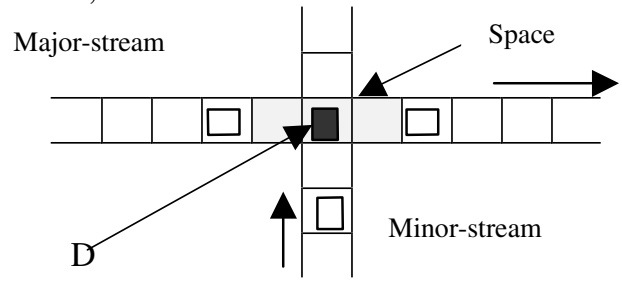

Fig. 1. Two-stream intersections with a rational driver D

A two-stream intersection (Fig. 1) is used to illustrate the driver interaction. Three cells give the minimum theoretical acceptable space for non-interruption of majorstream. Drivers are categorised as aggressive, rational and conservative.

A driver accepting a 3-cell space as the minimum acceptable space (MAP) is rational. A 2-cell space corresponds to aggressive behaviour. The effect is the blocking of the vehicle that has priority by the sub-rank vehicle. Conservative 
behaviour corresponds to $M A P \geq 4$ cells. Drivers are likely to prefer longer gaps for the more complex decision involved in turning, even though longer gaps are not required theoretically [17]. We assume therefore that most driver behaviour can be classified as rational or conservative. Probabilities associated with driver types sum to 1. Drivers are randomly reassigned to different categories with given probabilities at each time step, prior to checking whether the space meets the MAP. In this way, heterogeneity and inconsistency of driver behaviour are incorporated.

According to the rules of the road, a vehicle from a minor-stream has to obey a stop sign before it can enter an intersection. Our simulation ensures that all vehicles from the minor-stream will stop for at least one time-step (equal to 1 second). For minor-stream vehicles travelling straight-ahead or right-turning, two time-steps delay is allowed, in order to make a decision, (two major-streams are checked). We denote the time required as stop-sign-delay-time (SSDT). Thus, the follow-up time for a minor-stream in the simulation will be from 3 to 4 seconds, which agrees with the recommended follow-up time from observed data [16].

The main difference between our CA model and gap-acceptance models, in general, is that the critical gap in the gap-acceptance model and the MAP in our model have different temporal and spatial content, although both provide criteria for a driver to take action. For the gap-acceptance model, where the conflicting flow includes more than two streams, the gap is normally defined as the time taken for two vehicles from conflicting streams to pass through the path of the subject vehicle. Without distinguishing the direction that each vehicle comes from, the critical gap then has strong temporal meaning but is weak in spatial detail. However, in our model, the space required (in terms of different number of vacant cells required in each conflicting stream) is clearly specified so that temporal and spatial details are known precisely for each different movement indifferent streams, (details below), and the driver decision process is thus fully specified. Also, the critical gap is a fixed single value, whereas the MAP is a multi-value distribution corresponding to the distribution of driver behaviour.

\subsection{Minor-stream Vehicles: Straight-ahead $(S A)$ and Right-turning $(R T)$}

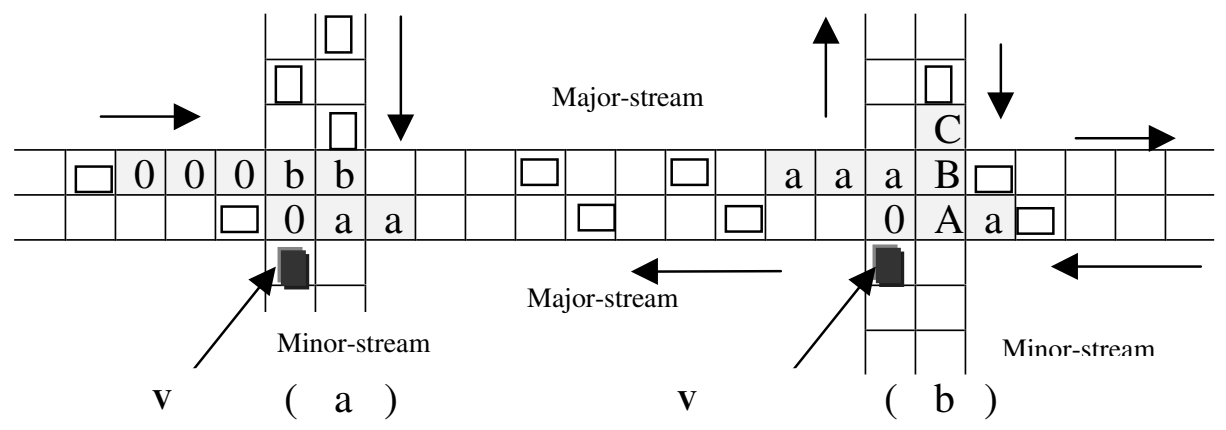

Fig. 2. A rational vehicle from a minor-stream moving (a) Straight-ahead (b) Right

For TWSC, conditions for the marked SA vehicle V to move into the intersection are illustrated. A rational driver needs to observe the 8 marked cells before s/he can drive 
into the intersection (Fig. 2a), whereas a conservative driver needs to check 10 cells. The marked cells, 0 , a, b and c, correspond to: " 0 " cell is vacant, "a" cell either vacant or occupied by a vehicle that will turn left, "b" cell not occupied by a right-turning vehicle and "c" cell either occupied by a right-turning vehicle or vacant.

In Fig. 2b, a SA or LT vehicle from the opposing minor-stream in cell marked "c" also has priority over the RT vehicle $\mathrm{V}$ from the given minor-stream according to the rules of the road. However, priorities between minor-stream vehicles might not be distinct [16]. Drivers were observed to enter the intersection on a first-come, firstserved basis. The movement of a RT vehicle from a minor-stream does not need to consider opposing vehicles if one of the following conditions is met.

- The first cell in the opposing minor-stream is vacant

- A RT vehicle is the first vehicle in the opposing minor-stream

- The first vehicle in the opposing minor-stream arrives at a stop-line in less than SSDT

\subsection{Left-turning (LT) Vehicle from a Minor-stream and RT from a major stream}

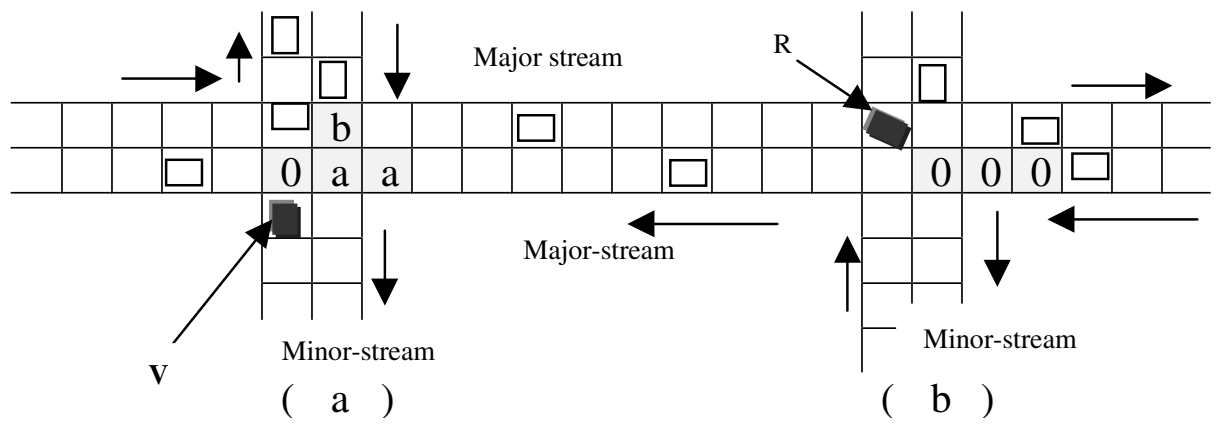

Fig. 3. A rational vehicle (a) LT from a minor-stream (b) RT from a major stream

Similar conditions apply to a driver turning left from a minor-stream (MiLT) and right turning from a major-stream (MaRT). A rational MiLT driver needs to check 4 marked cells before entering the intersection (Fig. 3a), whereas a MaRT vehicle R needs to check 3 marked cells (Fig. 3b).

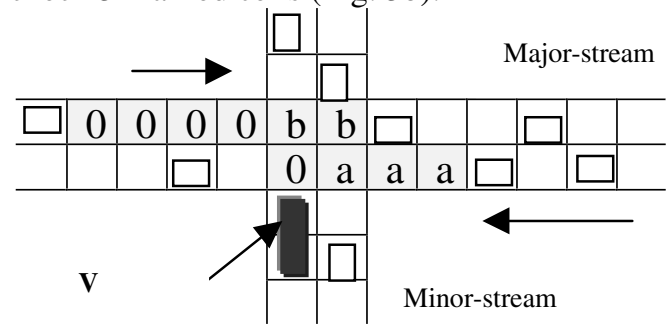

Fig. 4. A rational SA long vehicle from a minor-stream 


\subsection{Long Vehicles and Modified Intersections}

The case for a long vehicle has been considered briefly, based on occupation of two cells (Fig. 4). Crudely, rational movement for long vehicle through the intersection requires a check on 10 cells (as for a conservative car driver) in the simple model. Preliminary results indicate that long vehicles reduce throughput, as expected, but the impact of distribution assumptions has yet to be investigated. Flared minor-stream increases (e.g. [20]), can also be accommodated in our model, but have not been investigated to date.

\section{Model Implementation and Results}

Based on assumptions described, we studied performance, (capacity, time delay and queue-length) of a TWSC intersection under different values of traffic flow parameters, such as arrival rate (traffic volume) and turning rate (turning proportion). Experiments were carried out for 36,000 time-steps (equivalent to 10 hours) for a street-length of 100 cells on all approaches. All driver behaviour was assumed rational unless otherwise specified. Vehicles arrive according to a Poisson distribution, (where $\lambda \leq 0.5$ (equivalent to $1800 \mathrm{vph}$ ) in general for free flow). If all arriving vehicles pass the intersection without queueing, the flow rate $\lambda=0.1,0.2$, $0.3 \ldots$ equivalents are $360 \mathrm{vph}, 720 \mathrm{vph}, 1080 \mathrm{vph} .$. respectively.

\subsection{Capacity of a Minor-street}

When a RT or SA vehicle from a minor-street involves two major-streams, the capacity depends on their flow rates and configurations. In order to determine impact of different turning rates and different major-stream combinations, a TWSC intersection is studied, which contains only right-turning and left-turning vehicles in the minor-stream. All major-streams are assumed to have only SA vehicles. The total number of vehicles per hour in major-streams is assumed to be $1440 \mathrm{vph}$, which is split between the near-lane stream, (vehicles coming from the right), and far-lane stream, (vehicles coming from the left). Both left-turning-rate (LTR) and rightturning-rate (RTR) are varied. The differences in turning rates of the minor-stream can be expressed in terms of turning rate ratio (TRR =left-turning rate: right-turning rate). The difference in flow rates of the two major-streams can be expressed in terms of flow rate ratio $(\boldsymbol{F} \boldsymbol{R} \boldsymbol{R}=$ flow rate of near-lane stream: flow rate of far-lane stream $)$.

Table 1. Capacity of Minor-street for TRR and FRR

\begin{tabular}{|c|c|c|c|c|c|}
\hline \multirow{2}{*}{ TRR } & \multicolumn{5}{|c|}{ Capacity (vph) } \\
\cline { 2 - 6 } & \multicolumn{5}{|c|}{ FRR(=Flow rate of near lane : Flow rate of far lane ) } \\
\cline { 2 - 6 } & $1440: 0$ & $1080: 360$ & $720: 720$ & $360: 1080$ & $0: 1440$ \\
\hline $1: 0$ & 196 & 397 & 585 & 755 & 900 \\
\hline $0.75: 0.25$ & 193 & 363 & 483 & 527 & 415 \\
\hline $0.5: 0.5$ & 190 & 331 & 413 & 408 & 286 \\
\hline $0.25: 0.75$ & 183 & 308 & 361 & 286 & 180 \\
\hline $0: 1$ & 177 & 288 & 321 & & 217 \\
\hline
\end{tabular}


Table 1 indicates that both TRR and FRR affect capacity. TRR has been varied by increasing the number of right-turning vehicles in the minor-street. We find that the capacity of the minor-stream decreases in general when TRR decreases. However, this effect differs as FRR varies. In general, a vehicle manoeuvre, which requires conflicting streams to be crossed, leads to reduction in capacity. This is clearly illustrated, for example, in the final column of the table where far lane flow is heavy and right turning ratio is gradually increased. Similarly, a large percentage of minor stream vehicles joining a busy near-lane will be delayed and so on.

\subsection{Capacity of a Major-street}

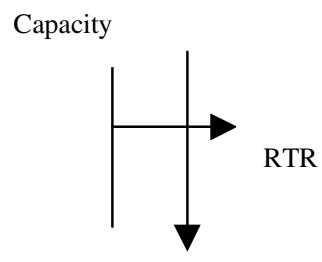

Conflicting Stream (CS)

(a)

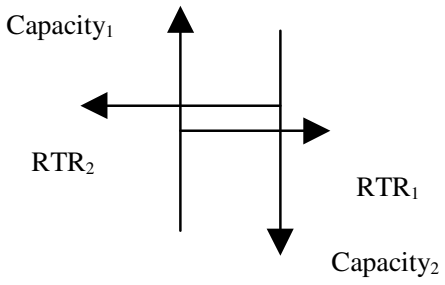

(b)

Fig. 5. Traffic configurations of shared lane on the major-streams

A right-turning vehicle in a shared major-street, where right-turning, straight-going and left-turning vehicles are on the one lane, can block SA and LT vehicles behind and in the same stream. Right-turning rates (RTR) of major-streams thus have great impact on capacities of major-streams. Two configurations have been studied (Fig. 5), with the analysis of major-street capacity following that of Chodur [27].

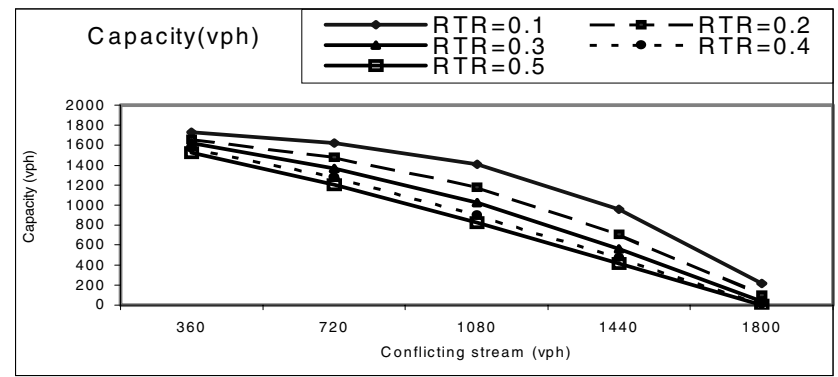

Fig. 6. Capacity of a major-stream as for Fig. 5a for rational driver behaviour

Table 2. Capacities and capacity ratio vs. right turning rate ratio

\begin{tabular}{|l|c|c|c|c|c|c|}
\hline & \multicolumn{7}{|c|}{$\mathrm{RTR}_{1}: \mathrm{RTR}_{2}$} \\
\cline { 2 - 7 } & $0.4: 0.1$ & $0.3: 0.1$ & $0.2: 0.1$ & $0.2: 0.2$ & $0.2: 0.3$ & $0.2: 0.4$ \\
\hline $\mathrm{Cap}_{1}: \mathrm{Cap}_{2}$ & $\sim 1: 4$ & $\sim 1: 3$ & $\sim 2: 1$ & $1: 1$ & $\sim 3: 2$ & $2: 1$ \\
\hline $\mathrm{Cap}_{1}(\mathrm{vph})$ & 413 & 541 & 758 & 1164 & 1373 & 1480 \\
\hline $\mathrm{Cap}_{2}$ (vph) & 1659 & 1616 & 1508 & 1164 & 911 & 740 \\
\hline
\end{tabular}


Fig. 6 shows unsurprisingly that the capacity of the major-stream declines rapidly with RTR as flow rate of the conflicting major-stream increases (Fig. 5a). Similarly, Table. 2 with RT vehicles in both major-streams yields a similar relation to that found form empirical study by Chodur [27], with Capacity $_{1}:$ Capacity $_{2}=R T R_{2}: R T R_{1}$.

\subsection{Queue-length and Delay}

Minor stream queue-length is found to depend on degree of saturation, $d$ (= flow rate/capacity) and also on arrival and turning rates of major and minor streams. For example, for LTR: SAR: RTR =0.2:0.7:0.1 and $F R R=0.15: 0.15$ on the major stream even a low arrival rate on the minor road leads to rapid queue build-up. Thus, for $d$ $=0.9(\lambda \cong 0.13)$ say on the minor approach, queue length builds to 34 cells with maximum delay time of 89 seconds. However, $50 \%$ of driver experience delay $<18$ seconds.

\subsection{Driver Behaviour}

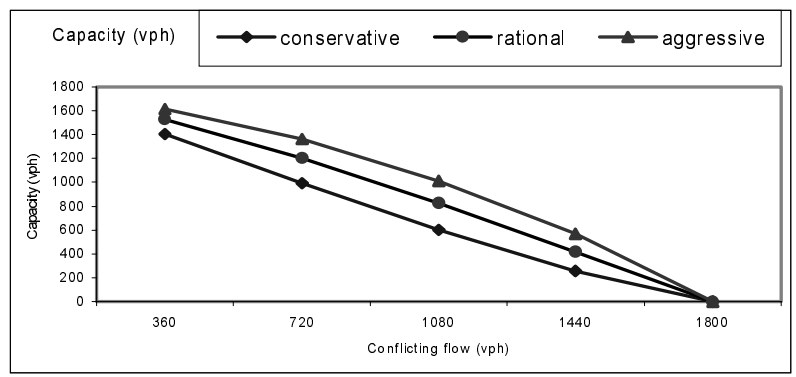

Fig. 7. Capacity of major-stream for Fig. 5a (aggressive, rational and conservative driver behaviour)

The effect of driver behaviour is to produce a series of capacity curves, similar to those for RTR (Fig. 5a). RTRs are fixed at 0.5 in the major stream (Fig. 7). Aggressive behaviour obviously increases the capacity, whereas conservative behaviour decreases it. The difference in capacity induced by driver behaviour is lessmarked for low and high level of conflicting flow (where capacity eventually $\rightarrow 0$ ), but is most noticeable for a conflicting flow rate of 1080vph -- up to $40 \%$ difference in capacity as a result of aggressive as opposed to conservative behaviour is observed for conflicting flow rates between 720 to $1440 \mathrm{vph}$.

Table.3 illustrates effects of different driver behaviour populations on capacity figures. In each scenario, turning rates and arrival rates are fixed. $\lambda_{1,2}$ or $3<0.5, \lambda_{4}=0.8$ $>0.5$ for approach 4 (minor-stream) only.

An approximate linear relationship is observed between capacities and driver behaviour ratio. Hence driver behaviour roughly determines the capacity of an unsignalised TWSC intersection. 
Table 3. Minor- stream capacity vs. driver behaviour

\begin{tabular}{|c|c|c|c|c|c|}
\hline \multirow{2}{*}{$\begin{array}{l}\text { Modelled } \\
\text { Scenarios }\end{array}$} & \multicolumn{5}{|c|}{ Driver Population ( Rational :Conservative) } \\
\cline { 2 - 6 } & $1: 0$ & $0.75: 0.25$ & $0.5: 0.5$ & $0.25: 0.75$ & $0: 1$ \\
\hline Scenario 1 & 518 & 492 & 464 & 435 & 406 \\
\hline Scenario 2 & 412 & 377 & 343 & 308 & 269 \\
\hline Scenario 3 & 527 & 504 & 482 & 461 & 437 \\
\hline
\end{tabular}

In general, our CA model agrees well with the validated results obtained from empirical and simulation studies e.g. [27].

\section{Summary}

A cellular automata model is used to simulate directly the interactions between drivers at a TWSC intersection using detailed space considerations. Heterogeneity and inconsistency of driver behaviour are also investigated and driver distribution is shown to have noticeable impact on capacity of major and minor streams, where a distribution, biased in favour of conservative driver behaviour, leads to a reduction.

The capacity of the minor-stream is shown to depend not only on the flow rates of major-streams, but also on flow rate ratios. The capacity of a minor-stream decreases when LTR decreases, but is less marked for FRR increases, which depend on increased flow rate of the near-lane.

Lacking real data, the distribution of driver behaviour is arbitrarily decided in the experiments, but the model can be used to investigate various assumptions and conditions of performance for TWSC intersections together with other features of urban traffic for which gap-acceptance models are less applicable.

\section{Acknowledgement}

We should like to acknowledge useful discussions with Zong Tian, Texas Transportation Institute, U.S.A.

\section{References}

1. Kimber, R. M.: The Traffic Capacity of Roundabouts. Transport and Road Research Laboratory Report 942, TRRL, Crowthorne, England (1980)

2. Brilon, W., Wu N., Bondzio, L.: Unsignalised Intersections in Germany-A State of the Art 1997. In: Kyte M. (ed.): Proceedings of Third International Symposium on Intersections without Traffic Signals. Portland, Oregon, U.S.A., University of Idaho (1997) 61-70

3. Tian, Z., Vandehey, M., Robinson, B. M., Kittelson, W. Kyte, M., Troutbeck, R., Brilon, W., Wu, N.: Implementing the maximum likelihood methodology to measure a driver's critical gap, Transportation Research A, Vol. 33. Elsevier (1999) 187-197

4. Nagel, K., Schreckenberg, M.: A cellular automaton model for freeway traffic. Journal de Physique I (France), 2: 2221 (1992)

5. Chopard, B., Dupuis, A., Luthi, P.: A cellular Automata model for urban traffic and its application to the city of Geneva. In: Wolf D. E. and Schreckenberg M.(eds.):Traffic and Granular Flow '97. World Scientific (1998) 153-168 
6. Wahle, J., Neubert, L., Esser J. , Schreckenberg, M.: A cellular automaton traffic flow model for online simulation of traffic. Parallel Computing, Vol. 27 (5). Elsevier (2001) 719-735

7. Robinson, B. W., Tian, Z.: Capacity and level of Service at Unsignalised Intersection: Final Report Volume1, Two-Way-Stop-Controlled Intersections. Access $28^{\text {th }}$ July 2001. http://books.nap.edu/books/nch005/html/R5.html\#pagetop (1997)

8. Troutbeck, R. J., Brilon, W.: Unsignalized Intersection Theory, Revised Traffic Flow Theory. http://www-cta.ornl.gov/cta/research/trb/CHAP8.PDF (1997)

9. Plank, A. W., Catchpole, E. A.: A General Capacity Formula for an Uncontrolled Intersection. Traffic Engineering Control. Vol. 25 (60) (1984) 327-329

10. Bottom, C. G., Ashworth R.: Factors affecting the variability of driver gap-acceptance behaviour. Ergonomics, Vol. 21 (1978) 721-734

11. Miller, A. J.: Nine Estimators for Gap-Acceptance Parameters in Traffic Flow and Transportation. In: G. Newell (ed.): Proceedings of the International Symposium on the Theory of Traffic Flow and Transportation, Berkeley, California. Elsevier (1972) 215-235

12. Troutbeck, R. J.: Estimating the Critical Acceptance Gap from Traffic Movements. Physical Infrastructure Centre, Queensland University of Technology. Research Report 92-1 (1992)

13. Brilon, W., Koening, R., Troutbeck, R.: Useful estimation procedures for critical gaps. In: Kyte M., (ed.): Proceedings of Third International Symposium on Intersections without Traffic Signals, Portland, Oregon, U.S.A., University of Idaho (1997)

14. Troutbeck, R. J.: Does gap acceptance theory adequately predict the capacity or a roundabout? In: Proceedings of the 12 ARRB (Australian Road Research Board) conference, Vol. 12, Part 4 (1985) 62-75

15. Hagring, O.: Estimation of critical gaps in two major streams. Transportation Research B, Vol. 34. Elsevier (2000) 293-313

16. Tian, Z., Troutbeck, R., Kyte, M., Brilon, W., Vandehey, M., Kittelson, W., Robinson, B. M.: A further investigation on critical gap and follow-up time Transportation Research Circular E-C108: $4^{\text {th }}$ International Symposium on Highway Capacity (2000) 397-408

17. Harwood, D. W., Mason, J. M., Robert, E. B.: Design policies for sight distance at the stop controlled intersections based on gap acceptance. Transportation Research A, Vol. 33. Elsevier (1999) 199-216

18. Troutbeck, R. J., Kako, S.: Limit priority merge at unsiginalized intersection. Transportation Research A, Vol. 33. Elsevier (1999) 291-304

19. Tracz, M., Gondek, S.: Use of Simulation to Analysis of Impedance Impact at Unsignalised Intersections. In: Brilon, W.(ed): Transportation Research Circular E-C108: $4^{\text {th }}$ International Symposium on Highway Capacity (2000) 471-483

20. Robinson, B. W., Tian, Z., Kittelson, W., Vandehey, M., Kyte, M., Wu, N., Brilon, W., Troutbeck, R. J.: Extension of theoretical capacity models to account for special conditions, Transportation Research A, Vol. 33. Elsevier (1999) 217-236

21. Wang, R., Ruskin, H.J.: Modeling traffic flow at a single-lane urban roundabout. In press, Computer Physics Communications (2002)

22. Chopard, B, Luthi, P. O., Queloz, P.-A.: Cellular Automata Model of Car Traffic in Twodimensional Street Networks, J. Phys. A, Vol. 29. Elsevier (1996) 2325-2336

23. Esser, J., Schreckenberg, M.: Microscopic Simulation of Urban Traffic Based on Cellular Automata. International Journal of Modern Physics C, Vol. 8, No. 5 (1997) 1025-1026

24. Yakawa, S., Kikuchi, M., Tadaki, S.: Dynamical Phase Transition in One-Dimensional Traffic Flow Model with Blockage, Journal of the Physical Society of Japan, Vol. 63, No. 10 (1994) 3609-3618.

25. Wang R., Ruskin H. J.: Modelling Traffic Flow at a Roundabout in Urban Networks, Working Paper CA-0601, School of Computer Applications, Dublin City University.http://www.compapp.dcu.ie/CA_Working_Papers/wp01.html\#0601 (2001)

26 Nagel, K.: Particle Hopping vs. Fluid-dynamic Models for Traffic Flow. In: Wolf D. E. et al. (eds.): Traffic and Granular Flow. World Scientific (1996) 41-56

27. Chodur, J.: Capacity of Unsignalized Urban Junctions. Transportation Research Circular EC108: $4^{\text {th }}$ International Symposium on Highway Capacity (2000) $357-368$ 